\title{
An outbreak of rubella at rural area of West Bengal, India in 2016
}

\author{
Dilip Kumar Biswas ${ }^{1}$, Rama Bhunia ${ }^{2}$, Doli Manna ${ }^{3}$, Anjan Chattaraj ${ }^{4}$
}

${ }^{1}$ Deputy Chief Medical Officer of Health-II, Purba Medinipur District; ${ }^{2}$ District Maternal and Child Health Officer, Howrah District; ${ }^{3}$ Senior Public Health Nurse, Sahid Matangini Block, Purba Medinipur district; Directorate of Health Services, Government of West

Bengal, India. ${ }^{4}$ Surveillance Medical Officer, National Polio Surveillance Project, Hooghly District, World Health Organization, India.

\section{Abstract}

Rubella is a contagious, generally mild viral infection that occurs most often in children and young adults. The infection in pregnant women may cause foetal death or congenital defects known as congenital rubella syndrome (CRS). Globally, the incidence of CRS was from 0.4-4.3/1000 live birth. The aims of the study were to (i) investigate outbreak to determine the magnitude of outbreak, and (ii) analyze outbreak in term of time, place and person distribution, and recommend preventive measures. We conducted investigation by house to house active case search at Dumra village of Purba Medinipur district, West Bengal; India between February and May' 2016. Epidemic curve was drawn to see the dynamic of outbreak and spot map was plotted to see the distribution of cases. Blood specimen was taken for serological test of virus. A total of 54 cases were identified; among those five cases were found positive for rubella (IgM). The attack rate (AR) among the women more 7.5\% (27/358) than men 7.0\% (27/388), with overall AR was 7.2\% (54/746). Maximum cases were found among age group of 5-9 years with AR was 8.0\% (23/286), followed by age group $0-4$ years with AR was 3.9\% (12/309). Median age was 8 years with rage from 0.6 to 33 years. Seventeen percent (9) cases were among female reproductive age (15-33 years). None of them were pregnant. Under five children were $33 \%(18 / 54)$ and $72 \%(13 / 18)$ were vaccinated with measles but none of them were vaccinated with rubella vaccine. All the patients were treated at home and at outpatient department. Outbreak of rubella was confirmed. We recommended introduction of rubella vaccine in National Immunization Schedule of India.

Keywords: Rubella, Outbreak, Rural Area, Preventive measure, India.

\section{Introduction}

Rubella is a mild febrile illness affecting the young children and adult is characterized by fever, macula-papular rash, cough, headache, conjunctivitis, arthralgia and lymphadenopathy. ${ }^{1}$ It is transmitted through droplets and direct contact of patients. It is also transmitted through placenta to foetus and causes serious effect on foetus called congenital rubella syndrome (CRS). ${ }^{2}$

The incidence of CRS globally from 0.4-4.3/1000 live birth. ${ }^{3}$ Most common congenital defect following CRS includes cataract, heart defect and impairment in hearing. It was reported that maximum chance of developing CRS was during first week to $11^{\text {th }}$ weeks of gestation. ${ }^{4}$ Vaccination against rubella virus is to prevent rubella infection during pregnancy and prevent CRS. Western and European countries eliminated measles and rubella by introducing vaccines. South East Asia countries fixed a target to eliminate rubella by $2020 .^{5}$

Rubella surveillance in India is integrated with measles surveillance programme. Annually, average 3263 cases were reported each year $(2012-2015)$ in India. ${ }^{6}$ But there is no appropriate data about rubella and CRS in India. ${ }^{7}$ The health workers informed about increase number of fever with rash cases at Dumra village of Purba Medinipur district, West Bengal India.

We investigated to confirm outbreak and to determine the magnitude of outbreak. We also analysed the outbreak in

\section{Practice Points}

- Outbreak of rubella increased along with measles outbreak.

- Attack rate among female were higher (7.5\%) than the men $(7.0 \%)$ with most of the cases were found among the age group of 5-9 years with AR was $8.0 \%$.

- Among cases $13 \%$ cases had out migratory history to measles endemic areas and $96 \%$ attended social gathering occurred at their locality.

- None of them were vaccinated with rubella vaccine.

- Specific recommendations:

- Introduction of rubella vaccine along with measles in national immunization programme.

- Recommend rubella vaccine to adolescent girls

- Awareness generation to community people regarding rubella.

terms of time, place and person distribution and recommended preventive measures.

Correspondence: Dr. Dilip Kumar Biswas, Welcome Housing, 228 Ashokegarh, Kolkata-108, West Bengal, India. Email: dilipbiswas29@gmail.com. 


\section{Materials and methods}

\section{Descriptive epidemiology}

Health workers of Dumra village, Sahid Matangini block reported to the Block Medical Officer of Health $(\mathrm{BMOH})$ and district surveillance officer about increased number of fever with rash cases in Dumra village during the first week of March' 2016. Block Medical Officer of Health (BMOH) primarily searched for clustering of cases in the locality. There were more than five similar cases in the village and the BMOH also informed Surveillance Medical officer (SMO) regarding the fever with rash cases. SMO is recruited by World Health Organization (WHO) for National Polio Surveillance Project (NPSP). The SMO visited the village and primarily diagnosed these cases as measles and launched first information report (FIR) to district and to the state. We reviewed the record of measles and rubella cases of the village Dumra for last five years and compared the data with present data to confirm outbreak.

\section{Study setting}

We conducted the investigation at Dumra village of Sahid Matangini block, district Purba Medinipur, West Bengal; India between February and May' 2016. The village had 2993 population. Population density of the village was 1782 /square Kilo meter whereas population density of the district Purba Medinipur was 1081 per square kilo meter. ${ }^{8}$

\section{Study design}

We conducted a cross sectional survey with active case search for measles and rubella by door to door survey. We searched cases as measles and rubella. We line listed all suspected cases with WHO case definition. ${ }^{9}$

\section{Case definition}

(i) Suspected measles case: Any person clinician suspects measles infection or any person with fever and macula-papular rash with cough, coryza or conjunctivitis.

(ii) Laboratory confirmed measles case: Presence of measles specific IgM antibodies.

(iii) Suspected rubella case: Any patient of any age in whom health worker suspects rubella is called as suspected rubella case. A health worker should suspect rubella case when a patient presents with fever, macula-papular rash; and cervical, sub-occipital or post auricular adenopathy or arthralgia/arthritis.

(iv) Laboratory-confirmed rubella case: Because of the difficulty of clinical diagnosis of rubella, laboratory confirmation is required. A laboratory confirmed case is a suspected case with a positive blood test for rubella specific IgM. The blood specimen would be obtained within 28 days after the onset of rash.

(v) Epidemiologically confirmed rubella case: A patient with a febrile rash which is linked epidemiologically to a laboratory confirmed rubella case.

From the above case definitions we made an operational case definition for health workers to conduct the survey as 'fever with macula-papular rash, arthralgia, cough and coryza, conjunctivitis and lyphadenopathy' any people resided in Dumra village during the study period. The date of appearance of fever or rash over the body was the date of onset of case.

Confirmed cases were defined who were laboratory confirmed for rubella IgM antibody. During outbreak investigation, if one or two cases were confirmed for measles or rubella or any other virus or bacteria or parasites, then no need to examine all suspected cases to detect such microorganism. So, all fever with macula-papular rash cases were line listed and checked for epidemiologically linkage with other cases were considered as cases for investigation. Epidemiologically linked was meant cases that were close contract with similar signs and symptoms within three weeks. Close contact was defined those who lived in the same houses, attended the same school, social gathering and played together with others.

\section{Data collection and analysis}

We trained the health workers to collect data. We constructed data collection format in local language in Bengali and again translated into English during data analysis. We collected information about age, sex, residence, date of onset, vaccination status, signs and symptoms of cases, migration history, housing, local festival or social gathering and treatment history of case. Measles immunization status was assessed by reviewing immunization card of the cases and verified the immunization status by examining immunization record. We considered complications and death as a sequel disease that occurred within 30 days of onset. We drew the spot map of the locality showing distribution of cases. We calculated attack rate by age and sex using census population of the village. We also drew the epidemic curve to show the dynamic of the outbreak. We analyzed data by using Epi-info and excel software.

\section{Laboratory investigations}

Five blood specimens of the suspected measles cases were taken and specimens were sent to the designated laboratory named 'Institute of Serology' 3 Kyd Street, Kolkata -700016, West Bengal, India for serological test maintaining cold chain of $2-8^{\circ}$ Celsius. This laboratory is accredited by 'World Health Organization'.

\section{Results}

Descriptive epidemiology

Total 54 cases were found at Dumra village of Purba Medinipur district during February to May 2016. Among them, five $(5 / 5)$ cases were identified as $\operatorname{IgM}$ antibody positive for rubella. But there was no rubella cases reported from this village during the last three years.

Attack rate (AR) among the women was more 7.5\% $(27 / 358)$ than the men $7.0 \%(27 / 388)$ with overall AR was $7.2 \%$ (54/746). Maximum cases were found among the age group of 5-9 years with AR was $8.0 \%(23 / 286)$, followed by $3.9 \%(12 / 309)$ age group was 0 to 4 years (Table 1).

Median age was 8 years with rage from 0.6 to 33 years. Seventeen percent (9) cases were among the female reproductive age (15-33years). None of them were pregnant. Thirty three percent $(18 / 54)$ of them were under five children and $72 \%(13 / 18)$ were vaccinated 
with measles but none of them were vaccinated with rubella vaccine. All the patients were treated at home and at outpatient department (OPD). None of them were died.

Among the total case patients, 54\% (28) had the family income less than Rupees 3000 per month and 96\% (52) of case patients visited social gathering (festival) held at their locality. Seven cases $(13 \%)$ had travel history to measles endemic areas and resided there for about six days and ten guests (19\%) stayed few days at their house of case patients. Most of the cases were student by occupation $78 \%$ (42) and Hindu by religion $98 \%$ (53) shown in (Table 2).

The epidemic curve showed that outbreak started on $16^{\text {th }}$ February' 2016 and ended on $2^{\text {nd }}$ May' 2016. There were several peaks in the epi-curve. Maximum ten cases were seen in the month of March and April' 2016 (Figure 1).

Spot map showed maximum three cases were found from one family and cases were distributed all over the village (Figure 2).

\section{Laboratory findings}

Using the passive surveillance system of rubella all measles negative cases were tested with rubella $\operatorname{IgM}$ antibody. All five blood specimens were found positive for rubella and declared as laboratory confirmed rubella cases.

\section{Clinical features and case management}

Almost all the patients developed rash (98\%) and fever $(85 \%)$. Cough and conjunctivitis developed $65 \%$ and $44 \%$ respectively. All the suspected and confirmed cases were treated at home and at OPD of nearby block primary health center. All cases were treated with vitamin A oil supplementation according to age. Home isolation of the patients was done.

\section{Discussion}

The outbreak was identified as rubella and this outbreak was primarily investigated as suspected measles outbreak. The study identified 54 cases and among them 5 cases was confirmed as IgM antibody positive for rubella. Women were affected more than men. Most of the cases were in the 5-9 years age group. None of the cases were vaccinated with rubella vaccine. There was no routine rubella surveillance and vaccination programme in India.

Result of this outbreak was compared with the study conducted at Chandigarh ${ }^{10}$ where maximum cases were in the age group of below ten, similar findings were observed in this study. In this study, there was no serious complication and no mortality. This finding indicated that rubella infection was mild in nature. Similar findings were reported in other studies in Himachal Pradesh ${ }^{11}$ and Chandigarh, India. ${ }^{12}$ Only $72 \%$ of the under five children were measles vaccinated in this village which was below the state average and district average. Measles vaccination among the under five children were needed to be improved. Poor coverage of measles vaccination reduced to circulating measles and rubella antibody in the community. There would be chance of rubella infection among the higher age group instead of children. For this reason, adolescent girls were susceptible to acquire paradoxical increase rubella infection, simultaneously leading to increase number congenital rubella syndrome. ${ }^{13}$ This would be addressed by strengthening routine immunization programme and introduction of rubella vaccine in the routine immunization schedule. Rubella is Togaviridae family and enters the body through respiratory tract. It is rapidly transmittable among people through crowded situation. ${ }^{14,15} \mathrm{~A}$ social gathering (festival) held at the locality where a large number people accumulated, and villagers might had acquired

Table 1: Attack rate of rubella cases by age and sex

\begin{tabular}{|c|c|c|c|}
\hline \multicolumn{1}{|c|}{ Characteristics } & Population & Attack Rate (\%) \\
\hline Age (Years) & 12 & 309 & 3.9 \\
\hline $0-4$ & 23 & 286 & 8.0 \\
\hline $5-9$ & 10 & 331 & 3.0 \\
\hline $10-14$ & 9 & 746 & 1.2 \\
\hline $15-33$ & 27 & 388 & 7.0 \\
\hline Gender & 27 & 358 & 7.5 \\
\hline Male & 54 & 746 & 7.2 \\
\hline Female &
\end{tabular}

Table 2: Socio-demographic status of rubella cases

\begin{tabular}{|l|l|c|}
\hline \multicolumn{2}{|c|}{ Socio-demographic status } & Cases (\%) (n=54) \\
\hline Family income (per month) & Up to Rupees (Rs:) 3000 & $28(54 \%)$ \\
\cline { 2 - 3 } & More than Rs: 3000 & $24(46 \%)$ \\
\hline Risk factors & Attended social gathering at the locality (Festivals) & $52(96 \%)$ \\
\hline & Travel to measles endemic areas & $7(13 \%)$ \\
\hline & $\begin{array}{l}\text { Guest attended their home and live with them }>3 \\
\text { days (in migration) }\end{array}$ & $10(19 \%)$ \\
\hline Occupation & Student & $42(78 \%)$ \\
\hline & House wife & $5(9 \%)$ \\
\hline Religion & Others & $7(13 \%)$ \\
\hline & Hindu & $53(98 \%)$ \\
\hline \hline
\end{tabular}

South East Asia Journal of Public Health 2017;7(1):17-22 


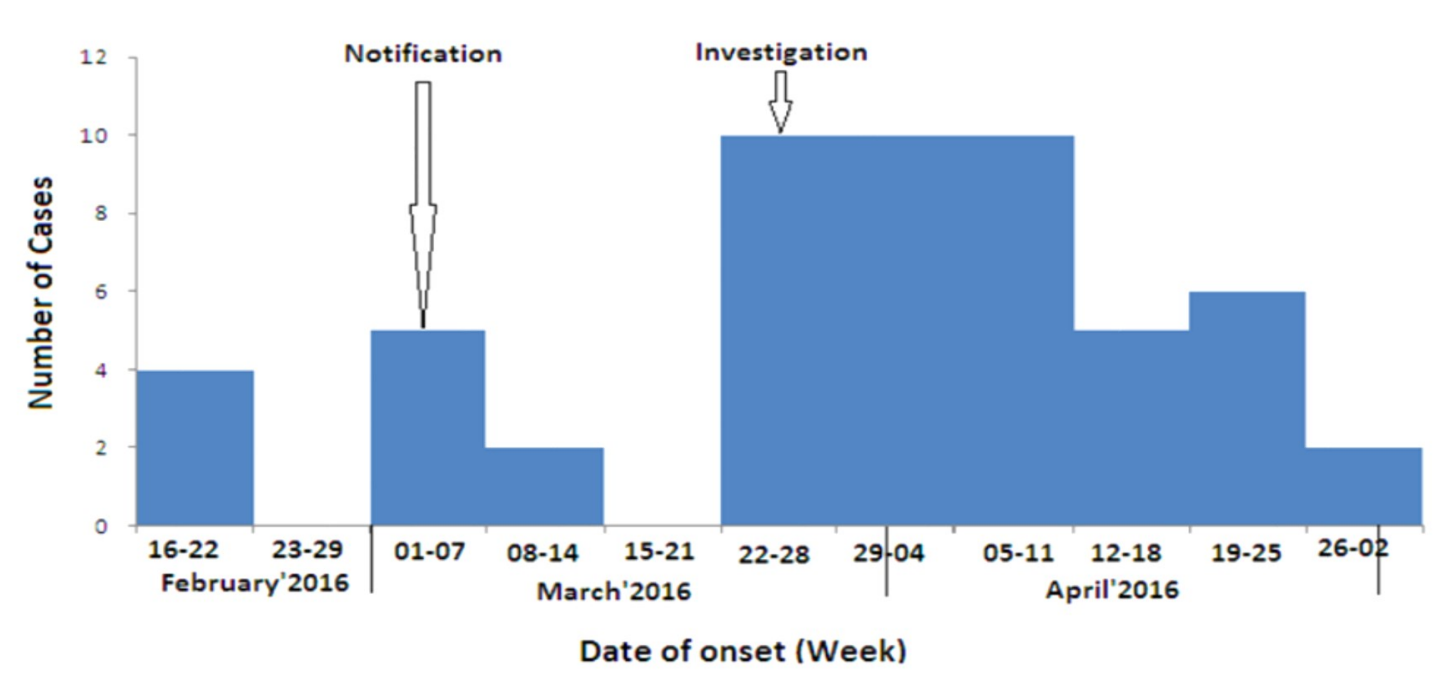

Figure 1: Epidemic curve of rubella outbreak $(n=54)$ of Dumra village

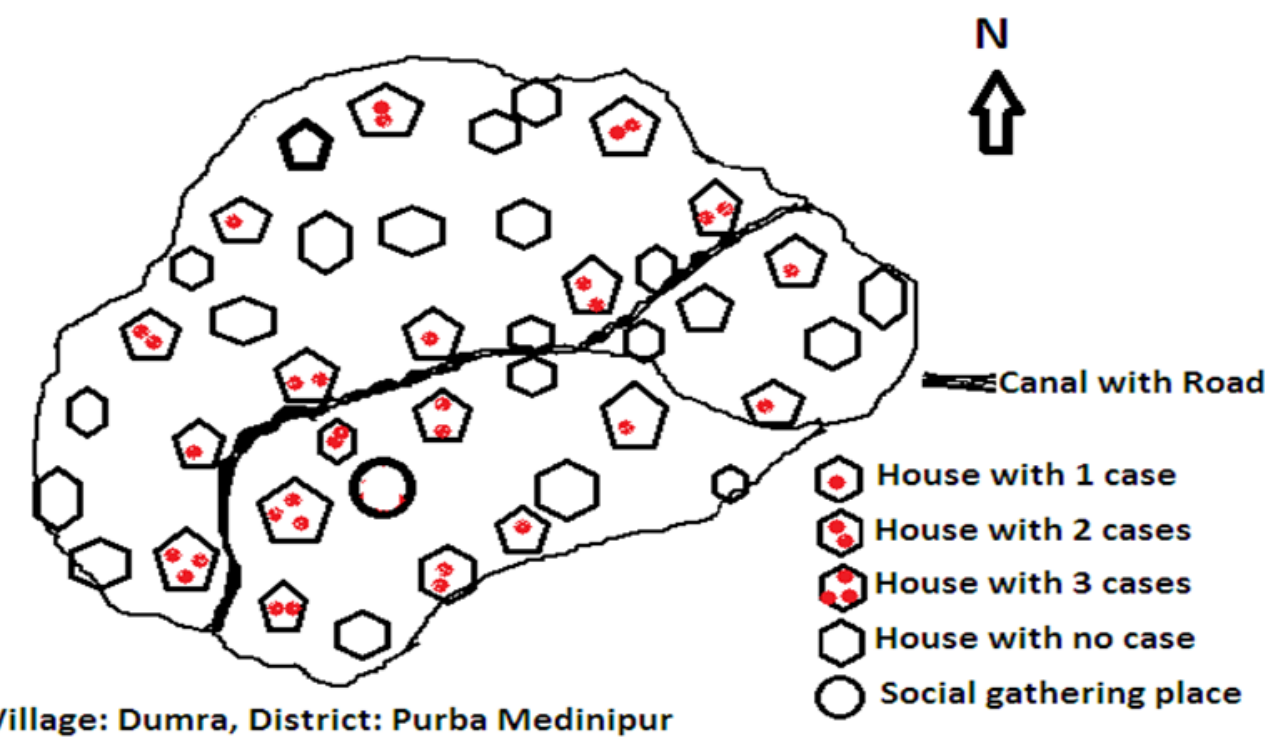

Figure 2: Spot map showing the distribution of rubella cases $(n=54)$ at Dumra village

rubella infection during this overcrowded condition. Studies at the United States ${ }^{16,17}$ suggested that migratory workers have higher susceptibility to spread rubella infection, because they were not immunized with rubella infection. But people of the United States were hardly affected with rubella infection, possibly vaccine induced antibody protect them. ${ }^{16,17}$ Here a number of case patients had migration history to measles endemic area and might had acquired virus from there and none of them were rubella vaccinated.

Rubella vaccination introduced in the United States in 1969 in National programme of immunization. In Western Pacific region, 11 countries also used rubella vaccine in national programme. Some Asian countries like Malaysia, Fiji, Japan, Hong Kong, Bahrain and Singapore are using this vaccine among the school children of age $11-15$ years to prevent CRS. ${ }^{18}$ To prevent CRS in India, rubella vaccine is needed to be introduced in the national immunization programme.
Though rubella is mild febrile illness, it affects on foetus and may cause abortion and congenital rubella syndromes. Congenital rubella syndromes are increasing in India.?

South Asian country, Kyrgyzstan introduced rubella vaccine in routine immunization schedule. ${ }^{19}$ National Polio Surveillance Project (NPSP) in India data showed that there was increased number of outbreak of rubella in West Bengal of India. There is also an increase trend of rubella outbreak in the district of Purba Medinipur (Table 3).

But there is no effective intervention programme and surveillance system for rubella and CRS in India. Considering the consequences of CRS, surveillance about rubella is to be strengthened and vaccine against rubella is needed to be introduced in the country. World Health Organization introduced rubella surveillance along with measles surveillance 
Table 3: Measles and rubella cases detected during outbreaks (2012-2016) in Purba Medinipur district, West Bengal

\begin{tabular}{|l|c|c|c|c|c|}
\hline \multirow{2}{*}{ Characteristics } & \multicolumn{5}{c|}{ Years } \\
\cline { 2 - 6 } & $\mathbf{2 0 1 2}$ & $\mathbf{2 0 1 3}$ & $\mathbf{2 0 1 4}$ & $\mathbf{2 0 1 5}$ & $\mathbf{2 0 1 6}$ (June) \\
\hline Total Measles Cases & 993 & 1101 & 1089 & 1107 & 849 \\
\hline Total Blood (Serum)Tested & 16 & 16 & 26 & 41 & 46 \\
\hline Number of Measles Confirmed & 10 & 10 & 10 & 12 & 12 \\
\hline Positivity Rate for Measles (\%) & 62.5 & 62.5 & 38.4 & 28.6 & 26.0 \\
\hline Number of Rubella Case confirmed & 6 & 0 & 16 & 14 & 14 \\
\hline Positivity Rate for Rubella (\%) & 37.5 & 0 & 61.5 & 34.1 & 30.4 \\
\hline
\end{tabular}

and strategies to introduce rubella vaccine along with measles vaccine to control the measles and rubella worldwide. ${ }^{13}$ As rubella is a mild febrile illness, people have different cultural belief about febrile rash and are reluctant to visit doctors. ${ }^{10}$ Extensive information education and communication (IEC) activity in the community would be helpful to identify outbreak early. In India, active rubella surveillance was not conducted and rubella vaccine was not introduced in the routine immunization programme except Delhi where rubella vaccine was introduced as Measles, Mumps and Rubella (MMR) vaccine among children $15-18$ month of age. ${ }^{20}$ Rubella vaccine is also commercially available in India.

\section{Limitations}

There might be a chance of recall bias due to outbreak was going more than two months. However, we collected information as early as possible to avoid the bias.

\section{Conclusion}

This was an outbreak of rubella occurred between the month of February and May 2016. Attack rate among the female were maximum than the men. Some of them were child bearing age, but nobody was pregnant. Five to 9 years old children were also affected. None of them were rubella vaccinated. All the cases were treated at home. Close contact and travel to endemic areas were related to rubella transmission. Number of rubella outbreak was increased than measles and this will be addressed by introduction of rubella vaccine in national immunization schedule.

\section{Recommendations}

We suggested introducing rubella vaccine in national immunization schedule along with measles vaccine and strengthening routine immunization and surveillance activity. We also suggested initiating awareness generation activity in the community, so that people could know the consequences of rubella infection and they will come forward for prevention and treatment.

\section{Competing interest}

The authors declared no potential conflicts of interest with respect to the research, authorship, and/or publication of this article.

\section{References}

1. Rubella. Oregon Public Health Division. Oregon.gov. March 2017. https:// public.health.oregon.gov/DiseasesConditions/ CommunicableDisease/

ReportingCommunicableDisease/ $\underline{\text { ReportingGuidelines/Documents/rubella.pdf }}$ (accessed July 2017)

2. Lanzieri TM, Segatto C, Siqueira MM, de Olivia Santos EC, Jin L, Prevots R. Burden of Congenital Rubella Syndrome after a community wide rubella outbreak, Rio Branco, Acre, Brazil, 2000-2001. Pediatr Infect Dis J 2003; 22(4):3239.

3. Robertson SE, Featherstone DA, Dobo MG, Hersh BS. Rubella and congenital rubella syndrome: global update. Pam Am Public Health 2003;14(5):306-15.

4. Miller E, Cradock Watson JE, Pollock TM. Consequences of confirmed maternal rubella at successive stages of pregnancy. Lancet 1982;2 (8302):781-4.

5. World Health Organization. Strategic Plan for Measles Elimination and Rubella and Congenital Rubella Syndrome Control in the South-East Asia Region, 2014-2020. New Delhi: WHO, 2015.

6. World Health Organisation. Rubella Reported Cases.http://apps.who.int/ immunization monitoring/globalsummary/ timeseries/tsincidencerubella.html (accessed July 2017)

7. Dewan P, Gupta P. Burden of congenital Rubella syndrome (CRS) in India: a systematic review. Indian Pediatr 2012;49(5):377-99.

8. Ministry of Home Affairs, India. Census of India 2011. www.censusindia.gov.in (accessed July 2017)

9. World Health Organization. WHO-recommended standard for surveillance of selected vaccine preventable diseases. 2003. WHO/V\&B/03.01. Geneva: WHO, 2003.

10. Madhanraj K, Sign N, Gupta M, Sign MP, Ratho RK. An Outbreak of Rubella in Chandigarh. Indian Pediatics 2014;51:872-4.

11. Gupta SN, Gupta NN. An outbreak of rubella in a hilly district of Kangra-Chamba, Himachal Pradesh, India, 2006. Indian $J$ Pediatr 2009;76:717-23.

12. Singh MP, Diddi K, Dogra S, Suri V, Varma S, Ratho RK. Institutional outbreak of rubella in a healthcare center in Chandigarh, North India. $J$ Med Virol 2010;82:341-4. 
13. WHO. Global measles and rubella strategic plan 2012-2020. Geneva: WHO, 2012.

14. Banatvala JE, Brown DW. Rubella. Lancet 2004; 3:363(9415):1127-37.

15. Chin J. Control of communicable diseases manual. $17^{\text {th }}$ edition. Washington: American Public Health Association, 2000.

16. Rangel MC, Sales RM, Valeriano EN. Rubella outbreaks among Hispanics in North Carolina: lesions learned from a field investigation. Ethn Dis 1999;9(2):230-6.

17. Danovaro-Holliday $\mathrm{MC}$, Le Baron $\mathrm{CW}$, Allensworth C, Raymond R, Borden TG, Murry $\mathrm{AB}$ et al. A large rubella outbreak with spread from the workplace to the community. JAMA 2000;6:284(21):2733-9.
18. Roberson SE, Cutts FT, Samuel R, Diaz Ortega JL. Control of rubella and congenital rubella syndrome in developing countries, Part 2: Vaccination against rubella. Bull World Health Organ 1997;75(1):69-80.

19. Gomber S, Arora SK, Das S, Ramchandran VG. Immune response to second dose of MMR vaccine in Indian Children. Indian $J$ Med Res 2011;134(3): 302-6.

20. Health Maternal and Child Health Community, Solution Exchange for the Maternal and Child health Community. Consolidated Reply. ftp:// solutionexchange-un.net.in/public/mch/cr/cr-semch-21100801-public.pdf (accessed April 2017) 